Conclusion GP auscultation has only moderate accuracy for diagnosing valvular heart disease in an unselected population, and the presence of an isolated murmur would not be a reliable indicator of valve disease. This study did not include patients with cardiovascular symptoms however, in whom the presence of a murmur may be more significant, and for whom echocardiography might be more appropriate.

Abstract 136 Table 2 The accuracy of cardiac auscultation in diagnosing significant VHD

The accuracy of cardiac auscultation in diagnosing significant VHD

\begin{tabular}{lllll} 
& & Murmur & & \\
& & Absent & Present & Total \\
\hline Echo Result & Negative & 148 & 66 & 214 \\
& & & & \\
& Positive & 21 & 16 & 37 \\
\hline Total & & 169 & 82 & 251 \\
\hline
\end{tabular}

Sensitivity $=0.43$

Specificity $=0.69$

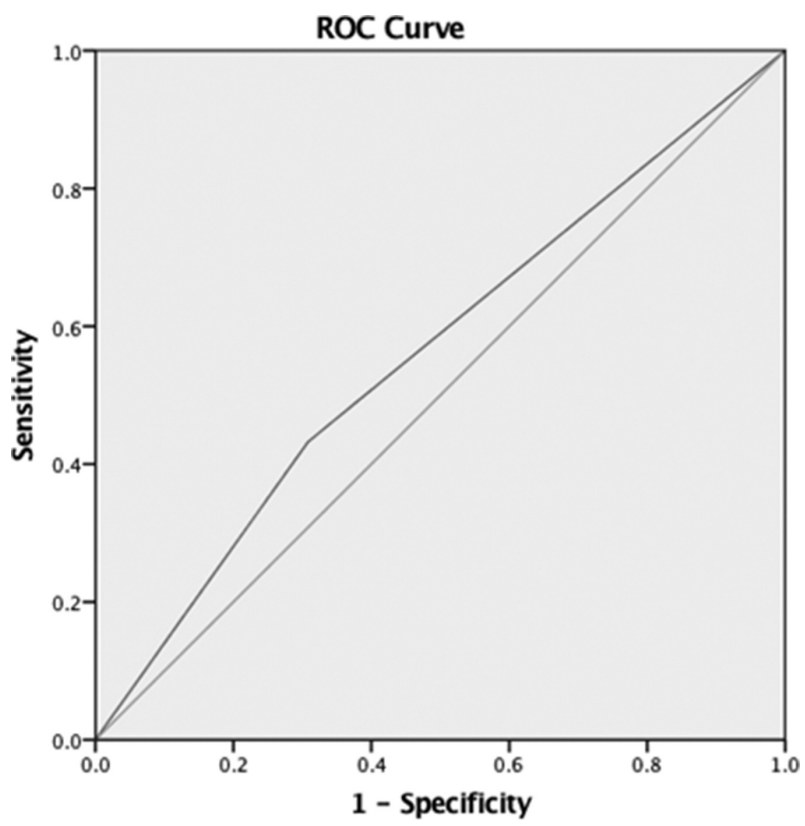

Diagonal segments are produced by ties.

Abstract 136 Figure 1 ROC curve for significant VHD Area under the curve $=0.56(95 \% \mathrm{Cl} 0.46-0.66)$

\section{ASSESSMENT OF PRE-OPERATIVE TRANSOESOPHAGEAL ECHOCARDIOGRAPHY GUIDELINE PREDICTORS OF MITRAL VALVE REPAIR SUCCESS WITH CURRENT SURGICAL PRACTICE}

Hanish Sall*, Lucy Ford, Thomas Mathew, Bara Erhayiem. NUH NHS Trust

10.1136/heartjnl-2017-311726.136

Introduction Mitral regurgitation (MR) is the second most common valvular heart disorder and severe MR brings with it a poorer prognosis. Successful mitral valve (MV) repair for prolapse has similar patient survival compared with expected outcomes. Precise definition of MV morphology is required to determine the complexity and feasibility of MV repair. Several echocardiographic parameters in international guidelines try and identify patients at risk of treatment failure. These are largely based on one study in 2002 where quadrangular resection and sliding plasty was the surgical technique of choice for the majority of MV prolapse. More modern surgical practice largely spares resection of valve tissue and employs neochordae/transfer techniques. This brings into question the applicability of previous imaging predictors for procedural success.

Hypothesis Are echocardiographic parameters in current recommendations accurate in predicting successful mitral valve repair?

Methods A retrospective study was carried out on all MV repairs undertaken at Nottingham University Hospitals NHS Trust between August 2015 and August 2016. Significant residual MR was defined as moderate or above. Echocardiographic predictors of suitability were taken from the European Association of Cardiovascular Imaging 2013 guidelines. Inclusion criteria: Pre-operative transoesophageal echocardiogram (TOE); post-operative baseline transthoracic echocardiogram (TTE) at 3-6 months. Exclusion criteria: lack of pre-op TOE or post-op TTE; previous cardiac surgery.

Results 52 patients underwent MV repair. Of these, 28 patients had complete imaging as set out by the inclusion criteria. Data presented as mean $\pm \mathrm{SD}$ unless otherwise stated. 4/ 28 (14\%) patients had significant residual MR. Table 1 shows demographics, pre-operative chamber characteristics, MV characteristics, operative technique and post-op MR characteristics between the two groups. There were no significant differences in demographics, cardiac chambers, MV or operative characteristics between patients with no significant residual MR and significant residual MR groups. No patients had quadrangular resection. The clinical characteristics of the 4 patients with significant residual MR are presented in table 2 .

Discussion In this small, single-centre, retrospective study, we found that none of the current echocardiographic predictors of suitability for MV repair were associated with presence of residual significant MR. Further prospective work is required to re-evaluate clinical and imaging data in predicting $\mathrm{MV}$ repair success with current surgical practice and improved imaging techniques.

\section{CAN PATIENT SAFETY AND OUTCOMES BE PRESERVED DURING LEARNING CURVE FOR AN INNOVATIVE SURGICAL PROCEDURE? THE EARLY RESULTS OF WOLVERHAMPTON AORTIC VALVE REPAIR PROGRAMME}

Maciej Matuszewski* ${ }^{*}$ Patrick Yiu, Antonella Meraglia, Giuseppe Bozzetti, Farhanda Ahmad. New Cross Hospital

\subsection{6/heartjnl-2017-311726.137}

Aim to evaluate early clinical and echocardiography outcomes of our recently initiated aortic valve repair (AV repair) programme.

Methods 26 patients were accepted for AV repair/replacement, starting in April 2014. The preoperative imaging was reviewed by our Cardiac MDT and by our proctor, Dr Emmanuel Lansac. The indications for surgery are listed in table 1 . 